\title{
Update in the diagnosis and management of acute pulmonary embolism for the non-respiratory physician
}

\author{
Authors: Sheila Ramjug ${ }^{A}$ and Gerrard Phillips ${ }^{B}$
}

An increasingly common scenario on the acute medical take is that of 'possible pulmonary embolism'. The aim of this article is to update the reader about the available clinical decision tools that can help to avoid the over investigation of such patients, as well as other tools that can support an outpatient management strategy in appropriate patient groups. The importance of risk stratification methodologies in acute pulmonary embolism management is emphasised. We address the evidence on the long-term risk of venous thromboembolism recurrence and show how this can be used to make decisions about duration of anticoagulation. Finally, we discuss a number of special scenarios, including the implications of incidentally discovered isolated subsegmental pulmonary embolus and the management of pulmonary embolus in malignancy and pregnancy.

\section{Introduction}

Chest pain in combination with dyspnoea and a raised D-dimer is a common presentation to the emergency department (ED) and the acute medial take. Pulmonary embolism (PE) is the third most common cause of acute cardiovascular presentation after myocardial infarction and stroke. ${ }^{1}$

The diagnostic dilemma in such clinical scenarios is how to prevent over investigation, while avoiding missing a significant PE. Over the last decade there has been an improvement in diagnostic and management strategies for PE, including better risk assessment, treatment and an increasing movement towards the safe management of some patients in the ambulatory/outpatient setting.

George is a 69-year-old, ex-smoker with a background of chronic obstructive pulmonary disease and diabetes, who presents to the ED with breathlessness and chest pain. George is currently clinically stable.

Interpreting the role of PE clinical tools, scores and D-dimer

The pulmonary embolism rule-out criteria (PERC) tool was developed to identify those patients with a low likelihood of PE

Authors: Aconsultant respiratory physician, Wythenshawe Hospital, Manchester, UK; B executive medical director, Federation of Royal Colleges of Physicians, London, UK and Dorset County Hospital, Dorchester, UK in whom unnecessary diagnostic work-up could be avoided. ${ }^{2}$ It comprises eight clinical variables (Box 1).

Two large prospective studies attempted to validate the use of the PERC rule in patients with a low likelihood of having a PE presenting to the ED. ${ }^{3,4}$ Both studies concluded that PE could be safely excluded in patients with low clinical probability of PE if they met all the criteria of the PERC rule. However, the external validity of these findings has been questioned due to the low overall prevalence of PE in the study populations. In one of the studies, which included 1,951 patients, the prevalence of PE was only $3.5 \%$ and $11 \mathrm{PEs}$ were missed using the PERC strategy. This issue was also noted by the National Institute for Health and Care Excellence (NICE) in their recent assessment of the utilisation of the PERC tool in their guidance on the management of venous thromboembolism (VTE). ${ }^{5}$

Despite George's stable clinical parameters, because of his age, he requires further diagnostic work-up according to the PERC tool.

Many studies have shown the superiority of clinicians' gestalt assessment when diagnosing PEs. Since this understandably

\section{Key points}

Venous thromboembolism is the third most common cause of acute cardiovascular presentations to the acute medical take.

Clinical decision tools used with measurement of D-dimer levels can support a rational and appropriate approach to the investigation of patients at risk of pulmonary embolism.

Assessment of pulmonary embolism severity allows patients categorised as low risk for mortality to be managed in the outpatient setting, provided suitable follow-up facilities are in place.

Assessment of risk factors for the pulmonary embolism event allows a rational approach to determining the duration of anticoagulation.

The incidence of chronic thromboembolic pulmonary hypertension is approximately $3 \%$.

KEYWORDS: venous thromboembolism, pulmonary embolism, chronic thromboembolic pulmonary hypertension

DOI: 10.7861/clinmed.2021-0666 


\section{Box 1. Pulmonary embolism rule-out criteria}

$>$ Age $<50$ years

$>$ Pulse $<100$ beats/minute

$>$ Arterial oxygen saturations $>94 \%$ (on room air)

$>$ No unilateral leg swelling

$>$ No haemoptysis

$>$ No recent trauma or surgery

$>$ No prior venous thromboembolism event

$>$ No exogenous oestrogen use

cannot be standardised, utilisation of clinical scores to assess the likelihood of PE is strongly recommended. The two most commonly used are the Wells Score and the Modified Geneva Score (Table 1). ${ }^{6,7}$

Clinical assessment of PE likelihood alone, either gestalt or using a validated score, has been shown to not be reliable enough to safely rule out PE in patients in whom this is suspected. Many studies have therefore sought to use a modification of Bayes' theorem in which the pre-test clinical probability of PE is combined with a subsequent test (D-dimer) result to produce a post-test likelihood of PE.

George's routine blood tests were unremarkable, but his high sensitivity troponin T was $45 \mathrm{ng} / \mathrm{L}$ (normal range $<14 \mathrm{ng} / \mathrm{L}$ ) and his D-dimer was elevated at $958 \mu \mathrm{g} / \mathrm{L}$ (normal range $<500 \mu \mathrm{g} / \mathrm{L}$ ).
Table 2. Years criteria

\section{Criteria}

Clinical sign of DVT eg swelling or pain

Haemoptysis

$\mathrm{PE}$ is the most likely diagnosis

\section{D-dimer adjusted cut-off range}

If no positive YEARS items, D-dimer cut off $>1,000 \mu \mathrm{g} / \mathrm{L}$

If $\geq 1$ positive criteria, D-dimer cut-off $>500 \mu \mathrm{g} / \mathrm{L}$

DVT = deep vein thrombosis; $\mathrm{PE}=$ pulmonary embolism

\section{Use of D-dimer in combination with clinical probability scores}

The 'YEARS' algorithm (Table 2) was designed as a simpler too to help assess the possibility of PE because algorithms such as the Wells Score are often either not used at all or not used well in busy EDs. ${ }^{8}$ The YEARS algorithm consists of three items from the Wells Score rule plus the D-dimer. This Dutch multicentre study included 3,465 consecutive patients. Thirteen per cent $(n=456)$ were diagnosed with a PE at baseline. Of the remaining cohort of 2,946 patients who had not been anticoagulated for other reasons, and in whom PE was ruled out at baseline, 18 patients were diagnosed with a symptomatic VTE event by 3 months' follow-up (0.61\%; 95\% confidence interval (CI) 0.36-0.96). The advantage of the YEARS algorithm over existing algorithms was a $14 \%$ reduction in the need

\section{Table 1. Clinical probability scores for pulmonary embolism: Wells and Modified Geneva Score}

\section{Wells clinical score for PE}

\section{Variable}

Previous PE or DVT

Heart rate $>100$ beats/minute

Surgery or immobilisation within the past 4 weeks 1.5

Haemoptysis

Active cancer

Clinical signs of DVT

Alternative diagnosis less likely than PE

\section{Clinical probability of PE based on calculated score}

Wells three-level probability

Low

Intermediate

High

\section{Wells two-level probability}

PE unlikely

PE likely

DVT = deep vein thrombosis; $\mathrm{GA}=$ general anaesthetic; $\mathrm{PE}=$ pulmonary embolism

\section{Modified Geneva Score for PE}

\section{Original Simplified Variable}

score score

$\begin{array}{ll}1.5 & 1 \\ 1.5 & 1\end{array}$

Age $>65$ years

Heart rate $<75$ beats/minute

Heart rate 75-94 beats/minute 3

Heart rate $>95$ beats/minute $\quad 5$

Surgery (under GA) or lower limb fracture in past 2 month

Haemoptysis

Active cancer

Unilateral leg pain 3

Unilateral leg swelling 4

Previous VTE

\section{Modified Geneva Score}

Low

$\leq 3$

Intermediate 4-10

2-6 N/A

High

$>11$


for computed tomography pulmonary angiography (CTPA) and, with that, reduced potential for radiation-induced harm and overdiagnosis.

Based on a 'YEARS' assessment, George would undergo further investigation if the $D$-dimer $>500 \mu \mathrm{g} / \mathrm{L}$ because he has one YEARS criterion ie PE was considered to be the most likely diagnosis (the chest $X$-ray was hyperinflated, with no obvious consolidation/collapse and electrocardiography was unremarkable).

\section{Age-adjusted D-dimer}

In patients over the age of 50 years, the use of age adjusted D-dimer may improve the diagnosis of PE. ${ }^{9}$ ADJUST-PE was a multi-centre prospective study of 3,346 patients, with a study PE prevalence of $19 \%$ in whom a sequential diagnostic strategy of clinical probability assessed using either the modified Geneva Score or the two-level Wells Score was followed by measurement of $\mathrm{D}$-dimer..$^{10}$ Of the 2,898 patients with a non-high or unlikely clinical probability, 817 had a D-dimer $<500 \mu \mathrm{g} / \mathrm{L}$ and 337 had a D-dimer between $500 \mu \mathrm{g} / \mathrm{L}$ and their age adjusted cut-off, defined as their age $\times 10$ in those older than 50 years. These patients did not have a CTPA, were not offered anticoagulation and were followed up for 3 months. Only 1/331 patients with a D-dimer $>500 \mu \mathrm{g} / \mathrm{L}$ but less than their age adjusted cut-off had a PE during the 3-month follow-up period, giving a failure rate for the age-adjusted cut-off of $0.3 \%(95 \%$ CI $0.1 \%-1.7 \%)$. In this study cohort, 766 patients were older than 75 years. Of these patients, 673 , had a low clinical probability of PE. By utilising an ageadjusted D-dimer score, 200 patients as opposed to 43 patients (using the standard D-dimer cut off of $500 \mu \mathrm{g} / \mathrm{L}$ ) from this subgroup had a PE diagnosis excluded, without any additional false negatives over a 3-month follow-up period.

George's age-adjusted D-dimer would be a value of $>690 \mu \mathrm{g} / \mathrm{L}$. Since his D-dimer was $958 \mu \mathrm{g} / \mathrm{L}$, a CTPA was requested.

\section{Risk stratification: inpatient or outpatient management?}

With intense and ever-increasing pressure on medical beds and on the availability of imaging modalities, it has become more common to consider which patients with possible low-risk PE might be managed on an outpatient basis. Such patients would be discharged from the ED/acute medical unit with appropriate overnight anticoagulation, but would be asked to return to an ambulatory unit for imaging within 24 hours. It is clearly important that selection of these patients is very carefully undertaken to avoid them coming to harm if they have had a PE.

A number of clinical risk assessment tools have been developed for this purpose. The tools that are recommended for use in the 2018 British Thoracic Society (BTS) guideline for outpatient management

Table 3. Clinical risk assessment tools to determine the suitability to manage a pulmonary embolism patient as an outpatient

\begin{tabular}{|c|c|c|c|c|c|}
\hline Hestia criteria & Score & PESI criteria & Score & sPESI criteria & Score \\
\hline Patient haemodynamically stable & Yes/no & Age & Age in years & Age $>80$ years & 1 \\
\hline Thrombolysis or embolectomy necessary & Yes/no & Male & 10 & & \\
\hline Active bleeding or high bleeding risk & Yes/no & Cancer & 30 & Cancer & 1 \\
\hline $\begin{array}{l}>24 \text { hours of oxygen to maintain } \\
\text { saturations }>90 \%\end{array}$ & Yes/no & Heart failure & 10 & $\begin{array}{l}\text { Chronic } \\
\text { cardiopulmonary } \\
\text { disease }\end{array}$ & 1 \\
\hline PE diagnoses during anticoagulation & Yes/no & Chronic lung disease & 10 & & \\
\hline $\begin{array}{l}\text { Severe pain requiring intravenous } \\
\text { analgesia }\end{array}$ & Yes/no & Pulse $\geq 110$ beats/minute & 20 & $\begin{array}{l}\text { Pulse } \geq 110 \text { beats } / \\
\text { minute }\end{array}$ & 1 \\
\hline $\begin{array}{l}\text { Medical or social reason for treatment in } \\
\text { hospital }\end{array}$ & Yes/no & $\begin{array}{l}\text { Systolic blood pressure } \\
<100 \mathrm{mmHg}\end{array}$ & 30 & $\begin{array}{l}\text { Systolic blood pressure } \\
<100 \mathrm{mmHg}\end{array}$ & 1 \\
\hline Creatinine clearance $<30 \mathrm{~mL} /$ minute & Yes/no & $\begin{array}{l}\text { Respiratory rate } \geq 30 \\
\text { breaths/minute }\end{array}$ & 20 & & \\
\hline Severe liver impairment & Yes/no & Temperature $<36^{\circ} \mathrm{C}$ & 20 & & \\
\hline Is the patient pregnant & Yes/no & Altered mental status & 60 & & \\
\hline $\begin{array}{l}\text { History of heparin-induced } \\
\text { thrombocytopenia }\end{array}$ & Yes/no & $\begin{array}{l}\text { Arterial blood oxygen } \\
\text { saturation }<90 \% \%^{a}\end{array}$ & 20 & $\begin{array}{l}\text { Arterial blood oxygen } \\
\text { saturation }<90 \% \%^{a}\end{array}$ & 1 \\
\hline Score risk & & Score risk & & Score risk & \\
\hline \multirow[t]{4}{*}{ Low } & All 'no' & I: Very low & $\leq 65$ & Low & 0 \\
\hline & & II: Low & $66-85$ & & \\
\hline & & III: Intermediate & $86-105$ & & \\
\hline & & IV: High & $106-125$ & & \\
\hline High & Any 'yes' & V: Very high & $\geq 126$ & High & $\geq 1$ \\
\hline
\end{tabular}

${ }^{a}$ With or without the administration of supplemental oxygen; PESI = pulmonary embolism severity score; SPESI = simplified pulmonary embolism severity score. 
of PE are the pulmonary embolism severity index (PESI), simplified PESI (SPESI) and Hestia score, the latter of which is particularly useful in those patients with underlying cancer (see Table 3). ${ }^{11-14}$

George has oxygen saturations of $95 \%$ on room air, respiratory rate of 20 breaths/minute, a clear chest to auscultation, heart rate of 90 beats/minute sinus rhythm, and stable blood pressure of $132 / 84 \mathrm{mmHg}$. His troponin was raised but there were no obvious ischaemic changes on electrocardiography. George's PESI score is 89. This would place George in the intermediate risk category, consequently he was admitted to hospital to await his CTPA.

George's CTPA demonstrated a large thrombus in the right distal main pulmonary artery extending into the upper lobar branch. There was also the possibility of a peripheral infarct, lymphadenopathy and a possible suspicious lesion in the right upper lobe. There was mild upper lobe predominant emphysema. When reviewing the heart, there was no straightening of the interventricular septum or evidence of right heart strain to suggest significant pulmonary hypertension.

\section{Assessment of pulmonary embolism severity}

To ensure appropriate management of PE patients, risk stratification of disease severity is required. A multivariate risk stratification tool for this purpose has been included in the European Society of Cardiology and European Respiratory Society (ESC/ERS) collaborative 2019 guideline 'diagnosis and management of acute PE'.15 This includes the use of clinical parameters, imaging and cardiac biomarkers to assess for right ventricular (RV) dysfunction, as well as underlying cardiorespiratory comorbidities (Fig 1).

George was classified as PESI class III and SPESI score 1. This, plus his raised troponin, placed him in the intermediate-high risk category. However, George spent an uneventful night in hospital after initially being anticoagulated with treatment of dose low-molecular weight heparin (LMWH): this option was utilised overnight as it is readily available with minimal interactions with other medications.

\section{Role of echocardiography in the acute setting}

Due to the high clinical demand on cardiorespiratory investigation services, it can be difficult to obtain echocardiography for inpatients with pulmonary embolism. It is, therefore, helpful to consider circumstances where an inpatient echocardiography is essential. These would include those patients too unstable to be transferred for a CTPA but where there is diagnostic uncertainty about the presence of PE as well as if there is suspicion of intra-cardiac clot on the CTPA, as this will have implications for ongoing management. ${ }^{17}$

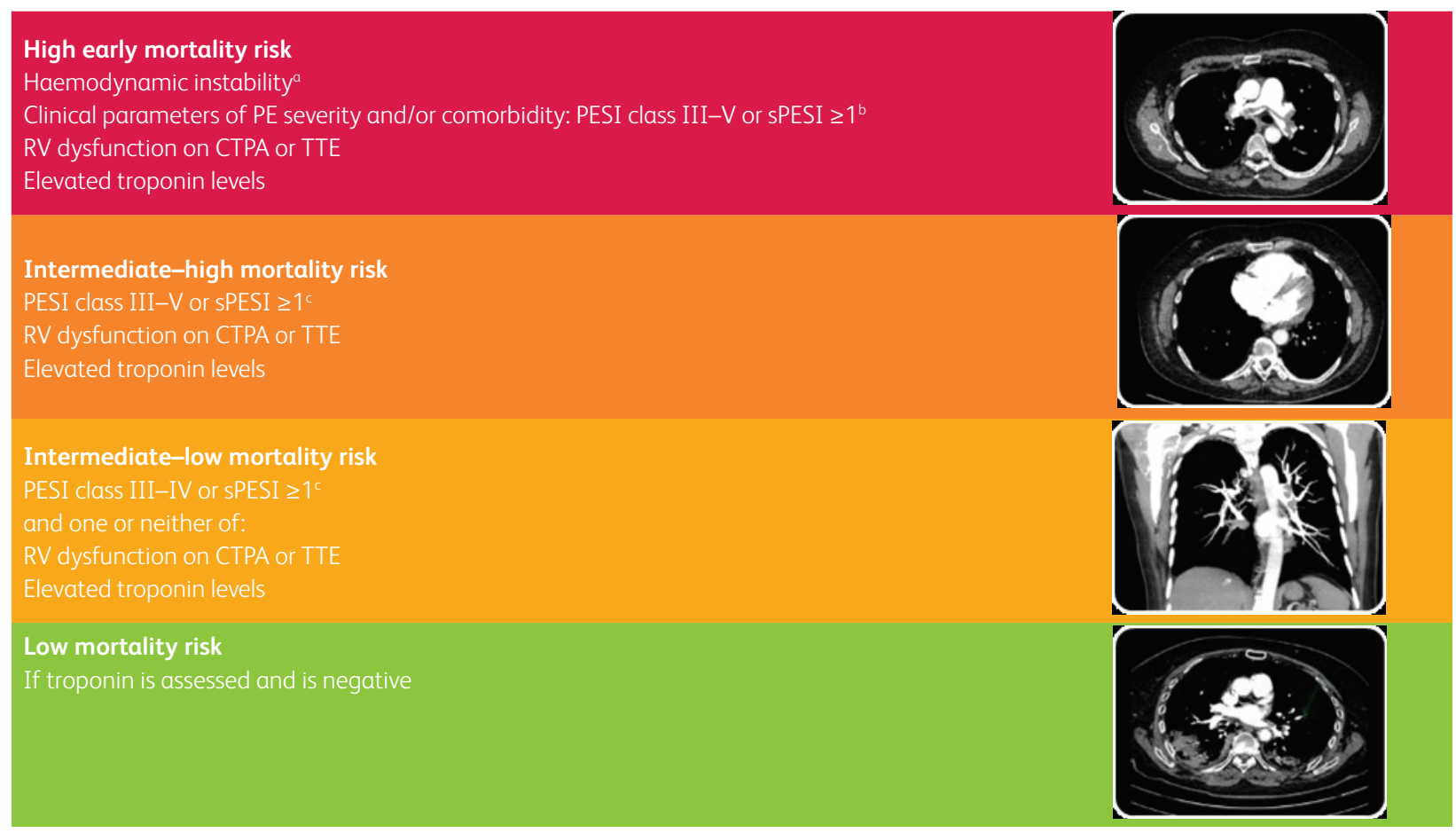

Fig 1. Demonstrating the severity of pulmonary embolism and associated risk of in-hospital / early (30-day) death adapted from ESC/ERS 2019 pulmonary embolism guidance. ${ }^{a H a e m o d y n a m i c ~ i n s t a b i l i t y ~=~ c a r d i a c ~ a r r e s t, ~ o b s t r u c t i v e ~ s h o c k, ~ p e r s i s t e n t ~ h y p o t e n s i o n ~(s y s t o l i c ~ b l o o d ~ p r e s s u r e ~} \leq 90 \mathrm{mmHg}$ or a systolic pressure drop $\geq 40 \mathrm{mmHg}$ for $>15$ minutes); 'In haemodynamic instability and evidence of PE on CTPA and/or evidence of RV dysfunction on echocardiography, calculation of PESI/sPESI or cardiac biomarker levels are not required to classify patients in this high-risk category; 'Signs of RV dysfunction on CTPA or echocardiography, as well as elevated troponin may be present despite a PESI ofI-II / sPESI of 0, the implications of these clinical findings are not fully understood, therefore, until this information is available, patients should be classified in the intermediate risk category: ${ }^{16}$ CTPA $=$ computed tomography pulmonary angiography; ESC/ERS = European Society of Cardiology and European Respiratory Society; PE = pulmonary embolism; PESI = pulmonary embolism severity score; RV = right ventricular; SPESI = simplified pulmonary embolism severity score; TTE = transthoracic echocardiography. 


\section{Role of half-dose thrombolysis}

Reperfusion therapy or thrombolysis with commonly recombinant tissue plasminogen activator is recommended in high mortality risk or haemodynamically unstable patients. In the older, comorbid PE patient this does carry an elevated risk of a life-threatening bleed. A meta-analysis from 2014 by Zhang et al suggested that those haemodynamically unstable patients managed with a reduced dose of thrombolysis had similar efficacy but fewer complications than for standard-dose thrombolysis. This metaanalysis included five randomised controlled trials: inclusion criteria was age $>18$ years and essentially haemodynamically massive PEs. This approach is, therefore, worth cautious consideration in appropriate elderly, comorbid high mortality risk PE patients. ${ }^{18}$

Nevertheless, the majority of admitted PE patients are haemodynamically stable. Those patients with evidence of RV dysfunction on CTPA or echocardiography, with elevated troponin levels and who are haemodynamically stable are categorised in the intermediate-high mortality risk group. Thrombolysis in this intermediate-high-risk group is controversial and not recommended as standard practice since, in the 2014 PETHIO trial, although the authors did demonstrate a reduction in haemodynamic instability or death in intermediate-high mortality risk PE patients, these thrombolysed patients had a substantially higher risk of major and intracranial bleeding. ${ }^{19}$

However, a number of small studies have suggested that a reduced-dose thrombolytic regimen may be as effective as fulldose thrombolysis in patients who are in the intermediate-high mortality risk category (ie haemodynamically stable but with evidence of RV dysfunction on echocardiography or CTPA and with a raised troponin) but with a lower risk of life-threatening bleeding. This would clearly be a preferrable option in such a cohort of more stable PE patients, especially in younger, less comorbid patients. PETHIO 3 is a randomised, placebo-controlled, double blind, multicentre, multinational trial that is currently recruiting to assess the efficacy and safety of a reduced dose of thrombolytic therapy in this intermediate-high-risk group (ClinicalTrials.gov identifier: NCT04430569).

\section{If there is no obvious risk factor, should I look for malignancy?}

Approximately $20 \%$ of PEs are associated with an underlying malignancy, and $20 \%$ of patients with cancers experience a VTE event at some point in the course of their disease. ${ }^{20}$

The current NICE guideline recommends against routine screening for an underlying malignancy (ie routine CT of the thorax, abdomen and pelvis in the absence of concerning clinical symptoms or signs) especially in those patients with a low mortality risk PE. ${ }^{5}$ It is strongly suggested, however, that a thorough clinical history and examination are undertaken, looking for red flag symptoms and signs and that, in men, the prostate-specific antigen is checked as well as ensuring that there are no overt signs of testicular pathology. In women, it is important to ensure a breast examination is completed and, if appropriate, encourage compliance with relevant screening programmes.

On the morning ward round, it transpired that George had noticed a new cough and some unexplained weight loss. Although he had ceased smoking when he was 65 years old, he had a 48 pack-year smoking history. He also had a family history of lung cancer, which might indicate that he is at increased risk of a lung malignancy. A repeat troponin that morning was normal. George was advised to start a direct oral anticoagulant (DOAC) after assessing his medications, renal function and

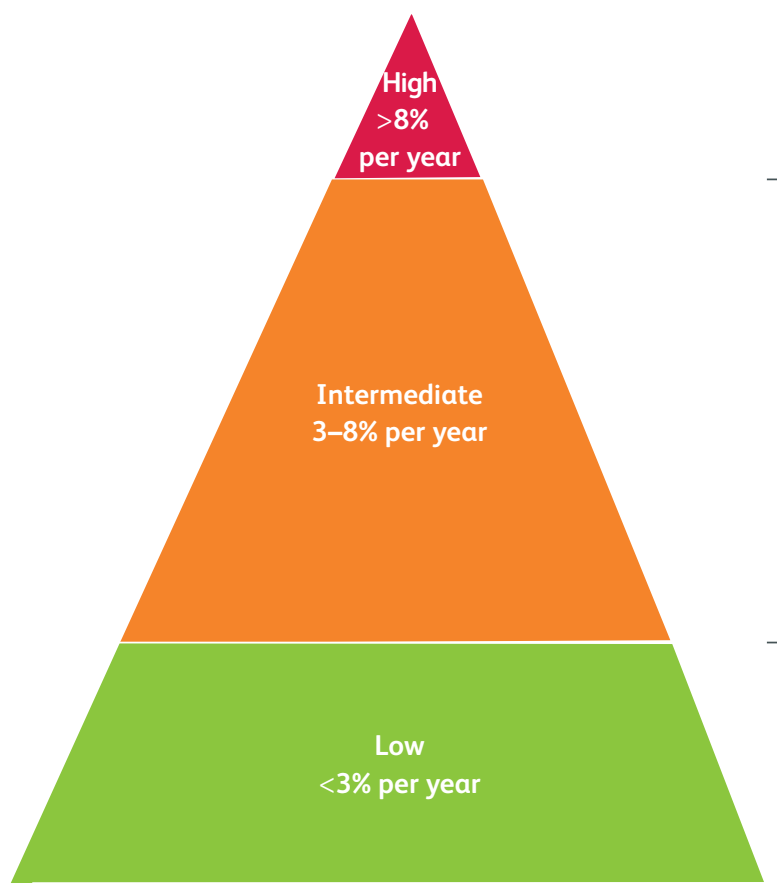
$>$ Active cancer
> One or more previous episodes of VTE in the absence of a major transient or reversible factor > Antiphospholipid antibody syndrome

\section{$>$ Transient/reversible factor associated with $\leq 10$-fold increase in VTE} risk

$>$ Minor surgery

$>$ Hospital admission $<3$ days with an acute illness

$>$ Confined to bed out of hospital $\geq 3$ days with an acute illness

$>$ Long-haul flight

$>$ Oestrogen therapy/contraception

$>$ Pregnancy or puerperium

\section{$>$ Non-malignant persistent risk factors}

$>$ Inflammatory bowel disease

$>$ Active autoimmune disease

$>$ No identifiable risk factor

$>$ Major transient/reversible factor associated with $>10$-fold increase in VTE risk

$>$ Surgery with general anaesthetic for $>30$ minutes

$>$ Confined to bed out of hospital $\geq 3$ days with an acute illness or exacerbation of a chronic illness

$>$ Trauma with a fracture

Fig 2. Categorisation of risk factors for VTEs based upon long-term risk of recurrence adapted from ESC/ERS acute pulmonary embolism guidance 2019. ${ }^{15}$ ESC/ERS = European Society of Cardiology and European Respiratory Society; VTE = venous thromboembolism. 
weight in addition to his bleeding risk; utilising a scoring tool such as VTE-bleed.

His Hestia Score was calculated, as was his sPESI score. His Hestia Score was low/negative and his sPESI score had not changed due to non-modifiable variables. As his troponin had also normalised, this too suggested that there was no contraindication to continued management in the outpatient setting with appropriate PE clinic follow-up as well as rapid referral to the lung cancer service.

\section{Special considerations}

\section{Use of direct oral anticoagulants in cancer}

Large trials have demonstrated the non-inferiority of edoxaban, rivaroxaban and apixaban in patients with cancer-associated thrombosis (CAT) compared with LMWH therapy. ${ }^{21}$ If there are no other contraindications to a DOAC, such as low renal function or platelet count, then DOACs should be strongly considered as an alternative to LMWH therapy in CAT. DOACs should, however, be avoided in luminal gastrointestinal or genitourinary malignancy due to the increased risk of bleeding.

\section{Incidental finding of subsegmental PE}

With modern developments in imaging, there is an increased incidence of reporting isolated single subsegmental PE. In the first instance, it is important to discuss this finding with the radiologist to ensure that it is not an artefact or a misdiagnosis. The VTE community is currently unsure of the clinical relevance of such a finding, especially if the patient is asymptomatic. To try to resolve this dilemma, there is a current ongoing multicentre, prospective, randomised controlled study (the STOPping Anticoagulation for isolated or incidental subsegmental Pulmonary Embolism (STOP-APE) trial), the aim of which is to ascertain whether withholding anticoagulation for a single isolated incidental subsegmental pulmonary embolism is superior to managing it with standard anti-coagulation in non-cancer patients (ClinicalTrials.gov Identifier: NCT04727437).

If there is, however, evidence of an isolated subsegmental PE then it is sensible to consider performing Doppler ultrasound (US) venography of the lower limbs to ensure that there is no concurrent deep-vein thrombosis (DVT), this being especially relevant in asymptomatic cancer patients, where such a finding would prompt therapeutic anticoagulation.

\section{Duration of anticoagulation in pulmonary embolism}

George was reviewed locally in the PE clinic 1 week after the initial VTE event. He was tolerating the DOAC therapy well and was compliant. A decision was taken to continue the DOAC therapy due to his now proven diagnosis of lung cancer, and this decision was to be reviewed at 4 weeks or earlier depending on his cancer therapy options ie to continue DOAC therapy or consider LMWH if George was receiving chemotherapy and/or immunotherapy.

Suspected PE in pregnancy

High pre-test probability or positive D-dimer and low-intermediate probability<smiles></smiles>

Treatment dose LMWH<smiles>CCC(C)(C)C</smiles>

CXR: if abnormal, consider alternative diagnosis

If symptoms and signs of a DVT:

compression proximal duplex ultrasound

(if signs of a pelvic thrombus, consider magnetic resonance venography)<smiles>C1CCCC1</smiles>

Proximal DVT present

CXR normal: arrange CTPA or low-dose perfusion scan CXR abnormal and continued concern of PE: arrange CTPA<smiles>C1CCC2CCCC2C1</smiles>

PE ruled out

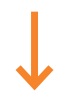

Positive or indeterminate Reviewed by radiologist
No proximal DVT present

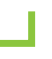

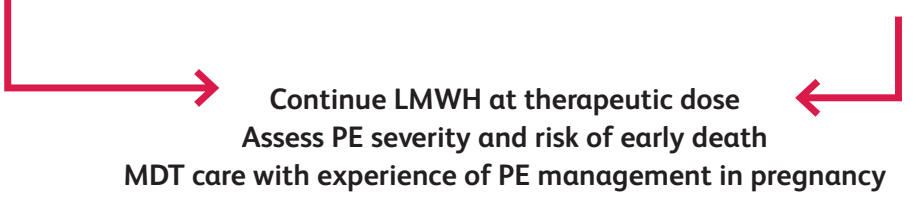

Fig 3. Suggested pathway for the diagnosis of pulmonary embolism in pregnancy adapted from ESC/ERS PE guidance. ${ }^{15}$ aEstimated amounts of radiation absorbed by fetus and maternal breast tissue vary between imaging modalities, low-dose perfusion scan vs CTPA, radiation dose to fetus $0.02-0.20$ vs $0.05-0.5 \mathrm{mGy}$, radiation dose to maternal breast tissue $0.16-0.5$ vs $3-10$ mGy; CTPA $=$ computed tomography pulmonary angiography; $\mathrm{CXR}=$ chest $\mathrm{X}$-ray; DVT = deep-vein thrombosis; ESC/ERS = European Society of Cardiology and European Respiratory Society; $\mathrm{LMWH}=$ low-molecular weight heparin; MDT = multidisciplinary team; $\mathrm{PE}=$ pulmonary embolism. 
All patients with an acute pulmonary embolism should be reviewed at 3 months with echocardiography to assess if there is any evidence of chronic thromboembolic pulmonary hypertension, which occurs in 3\% of patients. ${ }^{22}$ Suitable patients may be appropriate for consideration of pulmonary thromboendarterectomy. At this review point, one should also consider whether continued anticoagulation is indicated or not. This decision should be based upon the likelihood of a recurrent VTE event (Fig 2) and the bleeding risk. Those patients categorised as having a low VTE recurrence risk should cease anticoagulation at 3 months. In those patients with an intermediate risk of recurrence, long-term anticoagulation should be considered. It is recommended that those patients with a high likelihood of recurrence remain on anticoagulation life-long. In order to ensure that a patient is on the lowest dose of anticoagulation necessary to prevent a further VTE event, one strategy has suggested that, after 6 months of full anticoagulation, half-dose anticoagulation (in noncancer patients) should be considered. Studies have shown that this approach can be effective in preventing further VTE events. ${ }^{23}$

\section{Pregnancy and VTE}

Sadly, it is well established that PE is the leading cause of maternal mortality in the UK and that pregnancy and the puerperium increase the risk of a VTE event four- to sixfold. ${ }^{24}$

The 2019 ESC/ERS PE guideline suggests the use of D-dimer testing in pregnancy (Fig 3). The pregnancy-adjusted YEARS algorithm was a prospective study that safely ruled out and, therefore, avoided CTPA in $65 \%$ of patients in their first trimester and $32 \%$ in their third trimester. ${ }^{25}$ This clinical probability tool was adapted for pregnant women by including the use of compression ultrasonography of the pelvis/leg veins in women with clinical signs or symptoms of a DVT; if this was positive, a CTPA was not performed. If VTE is confirmed, LMWH is the therapy of choice.

\section{References}

1 Raskob GE, Angchaisuksiri P, Blanco AN et al. Thrombosis: a major contributor to global disease burden. Semin Thromb Hemost 2014;34:2363-71.

2 Kline JA, Mitchell AM, Kabrhel C, Richman PB, Courtney DM. Clinical criteria to prevent unnecessary diagnostic testing in emergency department patients with suspected pulmonary embolism. J Thromb Haemost 2004;2:1247-55.

3 Freund $\mathrm{Y}$, Cachanado M, Aubry A et al. Effect of the pulmonary embolism rule-out criteria on subsequent thromboembolic events among low-risk emergency department patients the PROPER randomized clinical trial. JAMA 2018;319:559-66.

4 Penaloza A, Soulié C, Moumneh T et al. Pulmonary embolism rule-out criteria (PERC) rule in European patients with low implicit clinical probability (PERCEPIC): a multicentre, prospective, observational study. Lancet Haematol 2017;4:e615-21.

5 National Institute for Health and Care Excellence. Venous thromboembolic diseases: diagnosis, management and thrombophilia testing: NICE guideline [NG158]. NICE, 2020:12-5.

6 Le Gal G, Righini M, Roy PM et al. Prediction of pulmonary embolism in the emergency department: The revised Geneva score. Ann Intern Med 2006;144:165-71.

7 Wells PS, Anderson DR, Rodger M et al. Derivation of a simple clinical model to categorize patients probability of pulmonary embolism: Increasing the models utility with the SimpliRED D-dimer. Thromb Haemost 2000;83:416-20.
8 van der Hulle T, Cheung WY, Kooij S et al. Simplified diagnostic management of suspected pulmonary embolism (the YEARS study): a prospective, multicentre, cohort study. Lancet 2017;390:289-97.

9 Righini M, Goehring C, Bounameaux H, Perrier A. Effects of age on the performance of common diagnostic tests for pulmonary embolism. Am J Med 2000;109:357-61.

10 Righini M, Van Es ], Den Exter PL et al. Age-adjusted D-dimer cutoff levels to rule out pulmonary embolism: The ADJUST-PE study. JAMA 2014;311:1117-24.

11 Howard LS, Barden S, Condliffe R et al. British Thoracic Society Guideline for the initial outpatient management of pulmonary embolism. Thorax 2018;73(Suppl 2):ii1-29.

12 Aujesky D, Obrosky DS, Stone RA et al. Derivation and validation of a prognostic model for pulmonary embolism. Am J Respir Crit Care Med 2005;172:1041-6.

13 Jiménez D, Kopecna D, Tapson V et al. Derivation and validation of multimarker prognostication for normotensive patients with acute symptomatic pulmonary embolism. Am J Respir Crit Care Med 2014;189:718-26.

14 Zondag W, Mos ICM, Creemers-Schild D et al. Outpatient treatment in patients with acute pulmonary embolism: The Hestia Study. J Thromb Haemost 2011;9:1500-7.

15 Konstantinides SV, Meyer G, Becattini. 2019 ESC Guidelines for the diagnosis and management of acute pulmonary embolism developed in collaboration with the European Respiratory Society (ERS). Eur Heart J 2020;41:543-603.

16 Barco S, Mahmoudpour SH, Planquette B et al. Prognostic value of right ventricular dysfunction or elevated cardiac biomarkers in patients with low-risk pulmonary embolism: A systematic review and meta-analysis. Eur Heart ] 2019;40:902-10A.

17 Condliffe R, Elliot CA, Hughes RJ et al. Management dilemmas in acute pulmonary embolism. Thorax 2014;69:174-80.

18 Zhang Z, Zhai ZG, Liang LR et al. Lower dosage of recombinant tissue-type plasminogen activator (rt-PA) in the treatment of acute pulmonary embolism: A systematic review and meta-analysis. Thromb Res 2014;133:357-63.

19 Meyer G, Vicaut E, Danays T et al. Fibrinolysis for patients with intermediate-risk pulmonary embolism. N Engl J Med 2014;370: 1402-11.

20 Sud R, Khorana AA. Cancer-associated thrombosis: risk factors, candidate biomarkers and a risk model. Thromb Res 2009;123 Suppl 4:S18-21.

21 Li A, Garcia DA, Lyman GH, Carrier M. Direct oral anticoagulant (DOAC) versus low-molecular-weight heparin $(\mathrm{LMWH})$ for treatment of cancer associated thrombosis (CAT): A systematic review and meta-analysis. Thromb Res 2019;173:158-63.

22 Delcroix M, Torbicki A, Gopalan D et al. ERS statement on chronic thromboembolic pulmonary hypertension. Eur Respir ] 2021;57:2002828.

23 Imberti D, Pomero F, Mastroiacovo D. Direct oral anticoagulants for extended treatment of venous thromboembolism: Insights from the EINSTEIN CHOICE study. Blood Transfus 2020;18:49-57.

24 Knight M, Tuffnell D, Kenyon S et al. Saving Lives, Improving Mothers' Care. Surveillance of maternal deaths in the UK 2011-13 and lessons learned to inform maternity care from the UK and Ireland Confidential Enquiries into Maternal Deaths and Morbidity 2009-13. MBRRACE-UK, 2015.

25 van der Pol LM, Tromeur C, Bistervels IM et al. Pregnancy-Adapted YEARS Algorithm for Diagnosis of Suspected Pulmonary Embolism. N Engl J Med 2019;380:1139-49.

Address for correspondence: Dr Sheila Ramjug, Wythenshawe Hospital, Southmoor Road, Wythenshawe, Manchester M23 9LT, UK.

Email: sheilaramjug@hotmail.com 